\title{
Translanguaging, recours aux langues et aux cultures de la classe autour de la littérature de jeunesse pour des publics allophones d'Ottawa (Canada) et de Montpellier (France) : opportunités et défis pour la classe
}

\author{
Carole Fleuret \\ cfleuret@uottawa.ca \\ UNIVERSITÉ D'OTTAWA \\ Nathalie Auger \\ nathalie.auger@univ-montp3.fr \\ UNIVERSITÉ PAUL-VALÉRY, MONTPELLIER III
}

\begin{abstract}
Résumé
Bien que vivant un contexte sociolinguistique bien différent, l'Ontario au Canada et le Languedoc-Roussillon en France partagent de nombreux points communs (idéologie de défense de la langue française, impératif de reconnaître la diversité scolaire) utiles à une réflexion sur l'enseignement aux élèves migrants, population de plus en plus grandissante sur les deux bords de l'Atlantique, et au translanguaging. Le manque de formation concernant le répertoire plurilingue de l'élève, les approches plurielles et les activités translangagières dans l'appropriation du français nous ont incitées à choisir des albums de jeunesse comportant des aspects interculturels afin de proposer à des enseignants sur nos deux sites d'utiliser ces artefacts. Après avoir formé un certain nombre d'enseignants à la mise en œuvre de l'interculturel et des albums de jeunesse en classe, nos résultats montrent que les enseignants ont des discours et attitudes contradictoires. Si l'album leur permet de s'autoriser et d'autoriser les langues et les cultures des élèves lors des séquences de classe, la majeure partie des activités translangagières proposées consistent à parler sur les langues et les cultures (sans inclure toutefois toujours celles des élèves) plutôt qu'à les utiliser comme une ressource à tout moment de la classe. Ces résultats nous amènent à proposer une redéfinition de la mise en œuvre des approches plurielles et des pratiques translangagières qui soient traduisibles dans les futures formations des enseignants.
\end{abstract}

Mots-clés : allophones, approches interculturelles, pratiques pédagogiques translangues, littérature de jeunesse 


\begin{abstract}
Although their sociolinguistic contexts are quite different, Canada's Ontario and France's Languedoc-Roussillon share many similarities (defence of the French language, necessity to acknowledge school diversity) useful for a reflection on the education of migrant students, whose number keeps rising on both sides of the Atlantic, and on translanguaging. The lack of training regarding the students' plurilingual repertoire, pluralistic approaches and translanguaging activities in French acquisition led us to choose children's books with intercultural aspects in order to encourage our teachers on both sites to use them. After offering teachers training on implementing an intercultural approach using children's books in class, we concluded that teachers have contradicting discourses and attitudes. While books let teachers allow the students' languages and cultures during the sessions, most of the suggested activities on translanguaging consist in talking about the languages and cultures (but without always including the students') instead of using them as a resource at any time in class. These results lead us to suggest a redefinition of the implementation of pluralistic approaches and translanguaging practices that would be "translatable" in future teacher training.
\end{abstract}

Key words: allophone population, intercultural approaches, pedagogical translanguaging practices, children's literature

\title{
Problématique
}

Le Canada accueille de plus en plus de nouveaux arrivants sur son territoire. En Ontario, dans ce contexte minoritaire francophone, environ 5\% des 13 millions d'habitants sont des immigrants (Statistique Canada, 2009). Bien évidemment, cette nouvelle réalité pose des défis pédagogiques indéniables et c'est la raison pour laquelle le ministère de l'Éducation de l'Ontario a créé et implanté deux programmes d'aide en français. Il nous faut préciser ou rappeler que le contexte sociolinguistique qui prévaut dans la province ontarienne à l'égard du français est un contexte minoritaire, ce qui rend complexe la place des autres langues dans la sphère scolaire.

Le premier, le Programme d'actualisation linguistique du français (ALF), ${ }^{1}$

\footnotetext{
${ }^{1}$ En 2002, le ministère de l'Éducation de l'Ontario lançait un programme-cadre en appui à des sous-groupes d'élèves ayant des besoins linguistiques et culturels distincts. Le programme ALF «s'adresse à l'élève qui a une connaissance limitée ou très limitée du français » (Ontario, 2002, p. 4). Contrairement au programme de 2002, qui préconisait l'appropriation de la culture francophone à titre de domaine d'étude en soi, le programme-cadre révisé de 2010 propose plutôt d'intégrer des référents culturels de la francophonie locale et mondiale à la pédagogie en vue «d'ancrer les apprentissages dans une réalité perceptible pour les élèves » (Ontario, 2010b, p. 5). Les contenus d'ap-
} 
qui vise à soutenir les élèves dans leur apprentissage du français de scolarisation, est conçu pour les élèves allophones, c'est-à-dire ceux qui ne parlent ni français, ni anglais, ni une langue des Premières Nations.

Le second, le Programme d'appui aux nouveaux arrivants (PANA) ${ }^{2}$ cible des élèves ayant été scolarisés dans un pays où le français est la langue d'enseignement ou d'administration publique. Certains de ces élèves récemment installés en Ontario ont connu une scolarisation différente de celle dispensée dans la province et ont subi des interruptions dans leur scolarité ou sont peu scolarisés (Ontario, 2010b, p. 5).

En France, l'immigration a toujours été un phénomène sociétal important. Et elle est fortement d'actualité. Les recensements de l'Institut national de la statistique et des études économiques (INSEE) estiment que près de 10\% de la population est « issue de l'immigration » et la «crise des migrants » est vive en Europe (INSEE, 2018). Si ces chiffres sont souvent controversés politiquement pour arguer de l'importance du phénomène afin de le présenter comme positif ou négatif dans l'espace médiatico-politique (Héran, 2017), ils n'en révèlent pas moins une situation indéniable, qui s'est accélérée ces quatre dernières décennies. Le Languedoc-Roussillon ${ }^{3}$ en est un exemple emblématique. Il est un

prentissage en matière d'identité culturelle liés à chacun des domaines sont les suivants :

- domaine A-La communication orale : L'élève doit reconnaître les référents culturels de la francophonie qu'il a entendus (ex. : radio, télévision) et intégrer ces référents dans ses communications orales.

- domaine $B$ - La lecture : L'élève doit reconnaître les référents culturels de la francophonie dans ses lectures, et les associer à ses repères culturels «pour élargir ainsi la gamme de référents qui définissent son identité » (Ontario, 2010b, p. 22).

- domaine $C$-L'écriture : Amener l'élève à inclure des référents culturels francophones dans ses écrits, des référents entendus ou rencontrés dans ses lectures.

${ }^{2}$ Le programme d'appui aux nouveaux arrivants (PANA) lancé en 2009 et révisé en 2010 (Ontario, 2009, 2010a) a pour but de répondre aux besoins de scolarisation « des élèves provenant de l'étranger et récemment installés en Ontario qui ne sont pas en mesure de suivre immédiatement le programme d'études ordinaire » (Ontario, 2010a, p. 3). Il vise particulièrement à aider les élèves qui arrivent de pays où le français est la langue d'enseignement ou d'administration publique, à se familiariser avec leur nouveau milieu, à s'initier à la société et à la culture canadiennes et à combler si nécessaire, des retards en communication orale, en lecture et en écriture. Le PANA est donc un programme de transition vers le programme d'études ordinaire qui est très similaire à l'ALF.

${ }^{3}$ Dans le cadre de la réforme territoriale, le Languedoc-Roussillon a fusionné avec la région Midi-Pyrénées en 2016 pour former la nouvelle région d'Occitanie. Du point de vue scolaire, l'Académie de Montpellier est toujours en charge de ce territoire et 
des territoires qui accueille le plus de migrants après l'Île-de-France. Situé en bordure de la Méditerranée, limitrophe de l'Espagne, cet espace est depuis le Moyen-Âge un lieu de rencontre entre le Nord et le Sud, facilement accessible par la terre ou la mer. Il est donc un territoire de premier choix pour la migration. Contrairement à l'Ontario, le Languedoc-Roussillon, tout comme le reste de la France, est un contexte francophone majoritaire. D'autre part, l'idéologie monolingue y est très forte, car l'unité linguistique du pays ne s'est pas effectuée sans mal après la Révolution française. Le pouvoir en place a donc décidé d'éradiquer à l'époque les nombreuses langues régionales jugées nuisibles à cette unité (Cerquiglini, 1999). L'idéologie monolingue est donc concomitante de l'émergence du concept d'État-nation en France. L'école, pilier et garante de l'unité du pays, est fortement marquée par cette idéologie du monolinguisme, sans arriver à s'en distancier ou à la considérer comme telle (Forlot, 2009). Cet état de fait est une donnée majeure pour cerner notre problématique qui vise à comprendre comment le translanguaging, lequel recouvrira, selon nous, les modalités de recours aux langues et aux cultures de la classe, représente à la fois des opportunités et des défis pour la classe. Car utiliser la diversité des langues dans un contexte fortement monolingue est une gageure même si elle est indéniablement une ressource de choix.

Du point de vue institutionnel, tout comme en Ontario, la France a mis en place un soutien scolaire spécifique pour les enfants migrants qui ne parlent pas français. Cette prise en charge a débuté dans les années 1970 au cours de ce que l'on appelle la «troisième vague d'immigration» en France, qui a commencé à la fin de la Seconde Guerre mondiale. Sans revenir sur les dispositifs de scolarisation de 1970 à nos jours (Auger, 2010; Auger et Cadet, 2016) qui se sont succédé les uns aux autres et qui rendent compte des différentes étapes dans la réflexion sur l'enseignement aux enfants migrants et sur les tensions entre les institutions et 1 . a recherche, le dispositif actuel prend la forme d'une unité pédagogique d'enseignement aux élèves allophones arrivants (UPE2A) ${ }^{4}$.

sert de point de référence pour discuter des questions de scolarisation, notamment des enfants migrants.

${ }^{4}$ Les Unités Pédagogiques pour élèves allophones arrivant (UPE2A) ont été créées à la suite de la circulaire de 2012 sur l'organisation et la scolarisation des élèves allophones en France par le Ministère de l'Éducation nationale (France, 2012).

Cette circulaire stipule que les élèves sont inscrits dans leur classe d'âge ordinaire mais qu'ils bénéficient d'un accueil en UPE2A à mi-temps pour soutenir le développement de leurs compétences en français. Dans le second degré, les élèves vont en classe ordinaire généralement en mathématiques, langues vivantes, arts plastiques et musique et en UPE2A le reste du temps. Ce dispositif est proposé pour une durée de 18 mois maximum.

Dans les faits, cette circulaire n'est pas mise en application partout, car en campagne 
Le principe est que la moitié du temps, les élèves sont avec les autres élèves de leur classe d'âge (notamment en mathématiques, arts plastiques, sport) et le reste du temps en UPE2A pour recevoir un soutien spécifique en français. Il faut noter que pour la première fois dans l'histoire de la dénomination de ces élèves, le terme allophone, suggéré par la recherche a été pris en compte dans l'acronyme du dispositif d'accueil et de scolarisation afin de reconnaître les répertoires plurilingues des enfants.

Si l'on considère à présent les résultats de ces dispositifs, on se rend compte qu'au Canada, bien que des services à l'appui en français soient dispensés dès l'arrivée des élèves dans le milieu scolaire, il semble que la réussite scolaire demeure fragile pour certains groupes ethnolinguistiques (Ledent, Aman, Garnet, Murdoch, Walters et McAndrew, 2013). En France, on partage le même constat même si les études sont rares (Schiff, 2012). Il est d'ailleurs symptomatique de constater que l'institution ne lance pas d'enquêtes statistiques sur la réussite scolaire des enfants migrants. Néanmoins, de nombreuses enquêtes qualitatives (Auger, 2010; Cadet, Mangiante et Laborde-Milac, 2009; Simon, Dompmartin-Normand, Galligani et Maire Sandoz, 2015; Goï, 2015) témoignent de la difficulté des enseignants à travailler avec ces élèves faute de formation spécifique, de suivi, de matériel. Sans conteste, les élèves sont dans une situation très fragile, tout comme en Ontario.

Bien que ces deux contextes sociolinguistiques diffèrent (minoritaire vs. majoritaire), ils se rapprochent par une volonté farouche de « défendre le français », ce qui ne facilite pas forcément a priori des pratiques de type translanguaging. Ces deux situations sont par ailleurs marquées par une histoire de l'immigration et des propositions de dispositifs de scolarisation qui manifestent des préoccupations conjointes. On peut donc légitimement les mettre en perspective et se questionner sur l'efficacité des pratiques mises en place dans les établissements scolaires au Canada et en France par rapport à la réussite scolaire des élèves nouveaux arrivants.

\section{La place de la diversité dans la salle de classe}

La réalité linguistique ontarienne, soit un contexte francophone minoritaire, rend complexe le statut accordé aux autres langues dans l'enceinte scolaire, et exacerbe le fait français (Fleuret, 2013). On voit alors émerger une totale dissonance entre les choix politiques canadiens qui prônent la diversité dans la pluralité, le multiculturalisme, et la réalité vécue par les élèves allophones. D'entrée de jeu, rappelons que l'école francophone en Ontario repose sur un

ou dans des écoles où il y a peu d'élèves allophones, les UPE2A ne sont pas créés systématiquement. Les enseignants composent donc avec ce public sans forcément être outillé pour cela. 
bilinguisme additif (McAndrew et Ciceri, 2003). Pour renforcer le fait français, les enseignants prennent appui sur le document intitulé : Une approche culturelle de l'enseignement pour l'appropriation de la culture dans les écoles de langue française de l'Ontario (Ontario, 2009). Un des piliers de cette approche concerne la construction identitaire qui se définit de la façon suivante (tiré du document de l'Association canadienne d'éducation de langue française, 2006) : «La construction identitaire est un processus hautement dynamique au cours duquel la personne se définit et se reconnaît par sa façon de réfléchir, d'agir et de vouloir dans les contextes sociaux et l'environnement naturel où

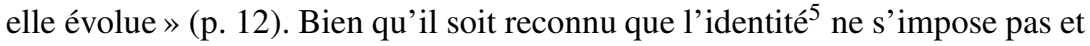
que l'élève puisse révéler une identité multiple, il demeure, malgré tout, une vision unidirectionnelle et figée vers le français : «l'élève doit se savoir valorisé en tant qu'usager de la langue française » (p. 18). Un élève nouvel arrivant va$\mathrm{t}$-il, pour reprendre les termes de la définition mise en exergue, se sentir dans un environnement naturel ? Va-t-il se reconnaître comme locuteur du français ? Bien que l'on prône dans le document et nous citons que : «l'école de langue française doit aussi encourager le plurilinguisme de manière à ce que l'attachement au français coexiste bien avec l'écoute et la prise en compte des besoins de la personne culturellement différente du fait de sa langue d'origine ou de son origine ethnique » (p. 19), il n'y a pas, à notre connaissance, d'espaces pour les autres locuteurs ni pour leurs cultures, puisque les seules dimensions culturelles retenues (collective et individuelle) visent la culture francophone de l'Ontario. Pourtant, comme l'ont remarqué Ferréol et Jucquois (2003) : « la diversité linguistique, pas plus que la diversité culturelle, n'entraînent inévitablement le conflit identitaire, à condition toutefois de se trouver inscrites dans le champ de la coopération sociale plutôt que dans celui de la compétition » (p. 160).

En 1994, Cardinal soulignait la prédominance de la culture française par rapport aux autres cultures. Elle ajoute que le concept de « Franco-Ontariens de souche », apparu en 1985 à la suite des positions très tranchées de l'Association canadienne-française de l'Ontario, est une façon claire de signifier qu'il y a des vrais francophones et d'autres qui le seraient moins. Cette posture de groupe dominé à groupe dominant laisse peu de place à la construction d'une francophonie ouverte sur le monde qui caractérise davantage la société plu-

\footnotetext{
${ }^{5}$ Cette définition opérationnelle met l'accent sur le côté dynamique du processus de construction, c'est-à-dire «se dire bilingue, francophone, bilingue anglo-dominant », etc., mais elle reste binaire et axée sur les deux langues officielles. Nous ne nous arrêterons pas ici à vouloir définir l'identité, concept ô combien polysémique, mais simplement rappeler que : "une relation inégalitaire entre ethnies ou nations, réduit les différences au bénéfice d'un seul système dominant (ethnocentrisme) » (Ferréol et Jucquois, 2003, p. 158).
} 
rielle que représente la province de l'Ontario aujourd'hui. Ainsi n'est-ce pas un hasard si, en 2008, Farmer et Labrie soulignaient déjà que malgré l'évidente hétérogénéité scolaire, la conception de l'identité culturelle restait homogène et que la mission historique de l'école ne s'était pas renouvelée. Encore aujourd'hui, pour souligner la diversité, non pas sans maladresse, les écoles font montre d'activités ponctuelles telles que la journée de l'art africain, le repas multiethnique, etc. qui sont en quelque sorte une focalisation sur des différences et qui construisent inévitablement une forme de folklorisation (Fleuret, Bangou et Ibrahim, 2013).

En France, l'idéologie monolingue en place, ainsi que le principe de laïcité qui se réifie dans le contexte de l'islamophobie grandissant et lié aux attentats ne facilite pas la prise en compte de l'altérité dans la salle de classe. La vision de la «liberté, égalité, fraternité » s'actualise dans un effacement des différences plus que dans leur reconnaissance. Pourtant, dans les années 1970, sous l'impulsion de chercheurs comme Abdallah-Pretceille (1995), Beacco (2000), Porcher (1998) ou Zarate (1986, 1993), une réflexion s'amorce sur l'interculturel et ses approches en didactique des langues. Ce domaine inclut même la notion de culture dans son traitement en parlant dorénavant de didactique des langues et des cultures (DDLC). Cette réflexion est sans surprise liée à l'émergence de la prise en compte des phénomènes de migration dans l'enseignement des langues. Le tournant communicatif des années 1980 ne désolidarisera plus le couple langue et culture qui se solidifiera dans l'approche actionnelle et continuera de le problématiser jusqu'à récemment, comme en témoignent les publications mentionnées ci-dessus.

Même si les enseignants de français langue étrangère ${ }^{6}$ qui suivent les formations universitaires de master sont formés aux approches interculturelles, rares sont ceux qui vont ensuite enseigner en français langue seconde aux enfants migrants dans les premiers et seconds degrés. En effet, en France, un concours est nécessaire pour enseigner à ces élèves et c'est une autre institution qui le prépare : l'ESPE (l'École supérieure du professorat des écoles). Les concours n'intègrent aucune matière interculturelle et une enquête que nous menons actuellement sur la formation à l'interculturel lors de l'année de formation suivant le concours indique que ces formations sont plus que sporadiques. On assiste alors, dans les classes, à ce que l'on peut appeler de la «pédagogie couscous » (Auger, 2010), c'est-à-dire que les enseignants, conscients de

\footnotetext{
${ }^{6}$ Les chercheurs européens emploient davantage le terme de langue étrangère pour faire référence à toute langue non maternelle n'étant pas la première langue d'appropriation linguistique; celle-ci étant apprise consciemment par l'apprenant, et non acquise de façon naturelle. Ce concept est plutôt peu utilisé au Canada; on lui préfèrera le terme de français langue seconde (Cuq, 2003).
} 
l'altérité de la classe et souhaitant la reconnaître pour ne pas apparaître discriminants, proposent ponctuellement une activité censée représenter la culture de l'autre : boire le thé, manger le couscous, etc. Les pratiques observées rejoignent donc de façon intéressante, une nouvelle fois, les observations menées en Ontario, qui sont aussi de l'ordre de la folklorisation (Fleuret et al., 2013). Dans les deux situations, nous sommes donc très loin des présupposés de la didactique interculturelle : on observe un manque indéniable de pratiques pédagogiques réellement plurilingues et pluriculturelles (Coste et ADEB, 2013), ce qui provoque des retards scolaires et des difficultés d'apprentissage.

\section{Langue seconde et littérature de jeunesse}

Pourtant, aujourd'hui, il est reconnu par les chercheurs en didactique des langues secondes (Auger, 2005, 2017a, 2017b; Coste et ADEB, 2013; Cummins, 2013; Fleuret, 2013; Fleuret et Thibeault, 2016; Hornberger, 2003), pour n'en citer que quelques-uns, l'apport indéniable des langues premières dans l'apprentissage de la langue de scolarisation. Ces dernières sont un levier sur lequel l'apprenant prend appui dans son apprentissage de la langue de scolarisation, ce qui favorise les transferts et l'acquisition de la nouvelle langue.

De plus, pour faire écho aux répertoires plurillitératiés des élèves (Moore et Sabatier, 2014) ou encore à leurs histoires de vie (Fleuret, sous presse), la littérature de jeunesse s'avère être un outil précieux à la prise en compte de la diversité. En effet, la multitude de choix, de formats et de contenus qu'elle propose permet la mise en place de pratiques pédagogiques fécondes.

On peut donc se questionner sur le type de pratiques pédagogiques implantées dans les écoles de l'Ontario et du Languedoc-Roussillon relativement à la prise en compte de la diversité dans la salle de classe.

\section{Cadre conceptuel}

Notre cadre conceptuel s'inscrit dans ce que Candelier (2008) appelle les approches plurielles. Cette dénomination regroupe des activités que nous nommerons translangagières et qui émanent de différents courants de recherches : l'intercompréhension entre les langues parentes (Blanche-Benveniste et Valli, 1997; Escudé et Janin, 2010) donc le fait de pouvoir inférer du sens dans une nouvelle langue à partir d'une langue ou de langues déjà connues; les approches interculturelles déjà évoquées ci-dessus ; l'éveil aux langues (Candelier, 2008; Hawkins, 1984; Kervran, 2006) qui vise à développer une conscience «méta» du fonctionnement des langues et du langage par l'observation de diverses langues ${ }^{7}$ et la didactique intégrée des langues (Cavalli, 2008; Roulet, 1980) qui met (selon les contextes) en contact des langues et des disci-

\footnotetext{
${ }^{7}$ Ces observations ne s'effectuent pas que sur la forme des langues, mais aussi sur le sens et les dimensions sociales du langage.
} 
plines scolaires. Ces approches dites plurielles renvoient donc à des situations de classe ou plus d'une langue est prise en compte («plusieurs» qui donnera «plurielles ») par opposition aux approches « singulières » d'une langue, qui ne font pas cas des autres langues du répertoire de l'apprenant.

Ces approches permettent de déconstruire la légitimité univoque de la langue de scolarisation qui occulte le répertoire langagier des élèves (Cuq, 2003). Cet aspect psycho-cognitif n'est pas négligeable, loin s'en faut. Si l'élève ne sent pas un locuteur légitime parce qu'il parle une ou des autres langues que le français, il aura des difficultés à avoir confiance en lui et en ses apprentissages. Par ailleurs, comme le soulignent García et Wei (2014) ces pratiques, que ces derniers qualifient de translangagières permettent les allers-retours cognitifs entre les langues de son répertoire (Auger, 2010). Ces activités sont de types « trans », permettant des va-et-vient entre diverses réalités, langagières et culturelles.

\section{La littérature de jeunesse}

L'intérêt du livre de jeunesse est sa complémentarité avec les activités langagières retenues par l'enseignant qui peuvent conduire à des activités translinguistiques, par l'entremise du contenu exploité. En effet, en fonction des choix opérés par l'enseignant (objet langagier à l'étude, prise en compte de la diversité, etc.), les albums plurilingues mettent en contact, sur les plans graphique et visuel, des scripts différents, faisant écho à la diversité (Fleuret, sous presse). Ils sensibilisent les élèves à la diversité des contextes, des langues et des cultures et permettent d'entrevoir le rapport à l'Autre par un prisme différent (Fleuret et Sabatier, 2019).

Comme le souligne Tauveron (2002, p. 26-30), dans une métaphore très éloquente, la lecture est une aire de jeu où l'initiation à la littérature convoque un plaisir esthétique, intellectuel et culturel. En d'autres mots, la littérature de jeunesse permet d'entendre la voix des élèves et leur rapport au monde. Autoriser cet espace intersubjectif pour reconnaître la singularité de chaque élève crée des ponts entre les élèves dans la reconnaissance des différences et l'ouverture à l'Autre. Ce cadre conceptuel nous permet donc d'observer comment se joue, dans nos deux contextes, la prise en compte des répertoires plurilingues et pluriculturels des élèves en classe, en relation avec des albums de jeunesse choisis pour leur mise en scène de diverses langues et cultures au cours de la narration.

Dans le cadre de la présente étude et en cohérence avec ce qui a été présenté, nos objectifs étaient les suivants :

- observer les interactions d'enseignants et d'élèves autour de l'album en 
ce qui concerne la diversité linguistique et culturelle (à la fois parler sur ${ }^{8}$ les langues et sur les cultures), après la formation reçue

- observer de quelles façons sont utilisées les langues et les cultures diverses pour parler, voire échanger autour d'elles et avec elles

\section{Méthodologie}

Pour notre recherche inter-site, nous avons privilégié une recherche mixte. Johnson et Onwuegbuzie (2004) soulignent que les études mixtes engendrent souvent des résultats supérieurs aux méthodes uniques, car elles permettent de décrire plus précisément l'objet à l'étude et le milieu dans lequel il évolue. Pour cet article, nous mettrons l'accent uniquement sur les enseignants.

Ils sont au nombre de 14 : six en France (trois pour le groupe expérimental et trois pour le groupe contrôle) et huit au Canada. Ils travaillent tous avec des élèves allophones. Trois des répondants du Canada appartiennent au groupe contrôle et les cinq autres au groupe expérimental.

\section{Le questionnaire}

Afin de connaître la façon dont est prise en compte la diversité dans la salle de classe, en Ontario (contexte minoritaire) et en Languedoc-Roussillon (contexte majoritaire), nous avons élaboré un questionnaire qui rend compte de leur rapport à la diversité et reprend les présupposés scientifiques que nous avons explicités supra. Certaines questions contiennent des ambiguités volontaires afin de nous assurer des réponses des participants au regard de leur cohérence. Le questionnaire (voir annexe 2) a été validé (contenu) par un expert, professeur en mesure et en évaluation. Il est composé de sept parties :

1. données socio-démographiques

2. connaissance des langues parlées par les enseignants et les contextes dans lesquelles elles ont été apprises

3. connaissance de chacune de ces langues en réception et en production

4. représentations des différentes langues selon une échelle de 1 à 5 , soit de la plus proche à la plus éloignée

5. compréhension de l'acquisition d'une langue seconde et des autres répertoires

6. connaissances des politiques et des programmes

7. pratiques pédagogiques et diversité culturelle

\footnotetext{
${ }^{8}$ Nous faisons le choix d'utiliser la préposition "sur" plutôt que "de" pour mettre l'accent sur le fait d'être «au-dessus de ... », donc d'aborder l'aspect méta.
} 
À travers ce questionnaire, ce que nous visons n'est pas une réponse en particulier, mais plutôt, à partir de l'ensemble des réponses, le profil émergeant de l'enseignant quant à ses expériences et ses répertoires didactiques.

\section{Formation des enseignants}

Le groupe expérimental a suivi une formation de trois jours sur la littérature de jeunesse et l'approche interculturelle. Les deux chercheures se sont déplacées, chacune leur tour, en France et au Canada, pour apporter leur expertise respective. Il s'agissait de donner des fondements théoriques sur l'interculturel et les albums de jeunesse et d'expliciter l'intérêt de ces pratiques tout en proposant des activités concrètes dont nous présenterons quelques exemples ci-dessous. Durant la dernière journée, une sélection de livres de littérature de jeunesse, abordant la diversité, a été présentée aux enseignants. Le choix des albums s'appuie sur la grille de Dupin de Saint-André et de Montesinos-Gelet (2011; vois annexe 1). Ils ont pu donc prendre le temps de les découvrir, d'en discuter et de choisir ceux avec lesquels ils aimeraient travailler (12 livres ont été choisis au total). Le choix des livres n'a pas été exactement le même des deux côtés de l'Atlantique bien qu'ils ne représentent qu'une petite quantité (trois); certains enseignants français trouvaient que certains livres étaient trop difficiles sur le plan langagier pour les proposer à leurs élèves.

\section{Observation participante (pendant 12 semaines)}

Nous avons également souhaité observer le milieu écologique dans lequel évoluaient les enseignants et de quelle façon, concrètement, ils prenaient en compte la diversité dans leur salle de classe. Berthiaume (2004) souligne qu'observer, c'est se rendre compte de ce qu'un individu perçoit, avec la plus grande objectivité possible, de la réalité qui l'entoure. C'est aussi, pour nous, une façon de trianguler nos données avec les autres instruments (questionnaire et entrevues) pour rendre compte d'incohérences potentielles.

Pendant 12 semaines, nous avons accompagné les enseignants du groupe expérimental dans une démarche didactique et interculturelle. Les figures 1 à 4 présentent quelques exemples d'aspects travaillés autour d'albums de littérature de jeunesse se référant aux approches plurielles et pouvant donner lieu à des activités translangagières/transculturelles, permettant des allers-retours entre les expériences diverses des élèves.

\section{Résultats et discussion}

Au regard de nos données préliminaires, nous pouvons dire sans ambages que les enseignants du groupe expérimental ont des conceptions diverses de la diversité. Pour ce faire, nous avons analysé les observations des classes de contrôle et expérimentales (prises de notes, parfois enregistrements vidéo en 


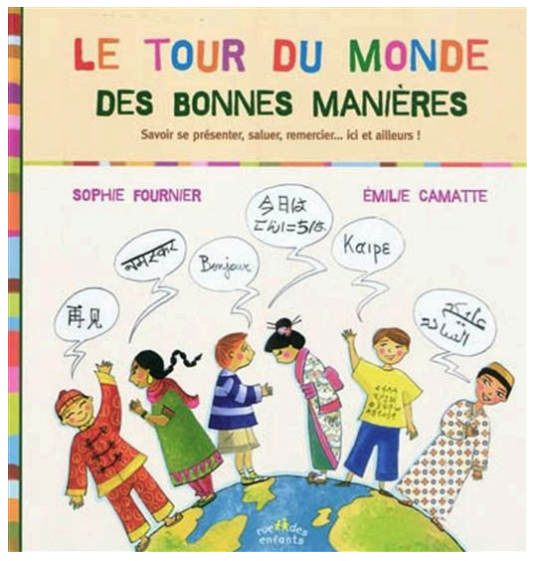

Séquence didactique : chercher les points de ressemblance et de différence dans divers contextes concernant les rituels d'interactions, comprendre qu'au-delà des spécificités, l'être humain a besoin de ces rituels pour entrer en relation, s'identifier, hiérarchiser; travail autour des écritures (scripts).

\section{Figure 1}

Le tour du monde des bonnes manières

(Fournier, 2011)

(C) Reproduite avec permission d'Éditions Rue des enfants/Sophie Fournier.

hubert Ben Kemoun - Bruno Heitz
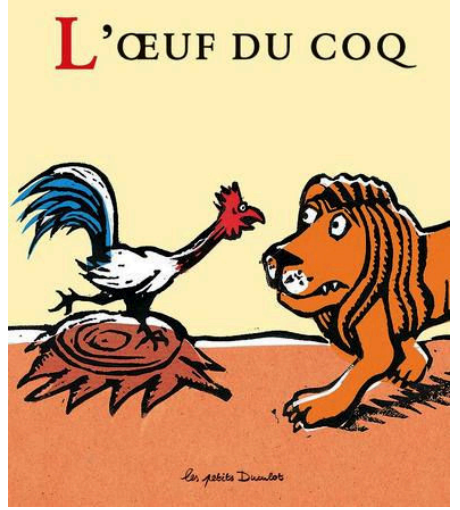

Séquence didactique : travail autour des emprunts langagiers et des langues dont ils sont issus ; sensibilitisation à l'évolution des langues, à leur dynamisme, souligner les coexistence des langues, etc.

\section{Figure 2}

L'œuf du coq (Kemoun, 2005)

(c) Reproduite avec permission de l'auteur Hubert ben Kemoun.

France pour les classes «expertes ») et les questionnaires et entrevues avec les enseignants. Nous présenterons maintenant quelques données dans leur ensemble.

\section{Dans les questionnaires : un consensus pour reconnaître les langues des élèves}

Tout d'abord, nous commencerons par explorer, dans les questionnaires, les discours des enseignants, qu'ils soient des groupes expérimental ou de contrôle, pour voir leurs attitudes par rapport aux langues et cultures des élèves. En effet, s'ils les rejettent ou les considèrent comme inutiles, les activités translangagières vont s'avérer impossibles à mettre en œuvre en classe. Nos résul- 


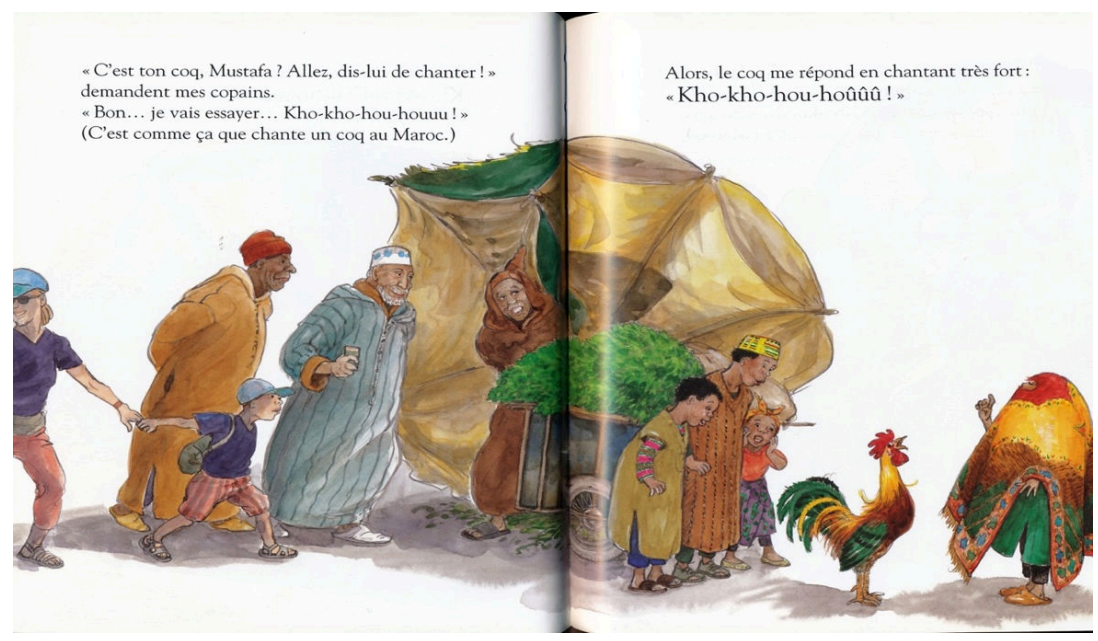

FIGURE 3

Le magasin de mon père (Ichikawa, 2007, p. 18-19)

(c) Reproduite avec permission d'Éditions l'École des loisirs/Satomi Ichikawa.

Séquence didactique : travail sur les onomatopées, leur origine et leur chaîne sonore, universalité et spécificité des onomatopées dans les langues et le langage. Travail intéressant sur les illustrations.

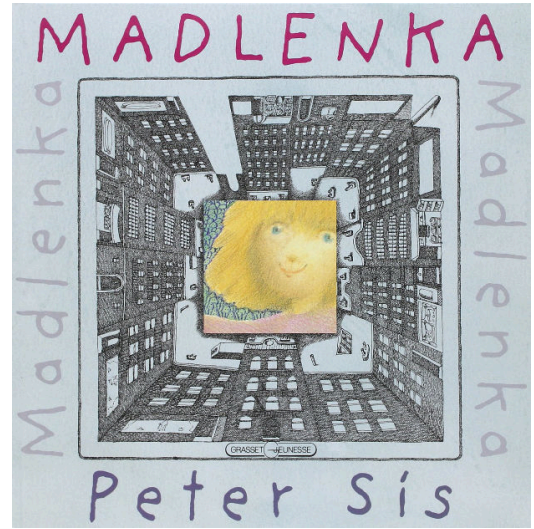

Séquence didactique : travail sur le prénom de l'héroïne qui se modifie en fonction du contexte et de la langue, spécificités phonologiques; travail sur les illustrations qui sont absolument époustouflantes dans cet album; travail sur l'expression idiomatique : «perdre une dent» et les pratiques sociales diverses qui en découlent.

\section{FIGURE 4}

Madlenka, (Sis, 2000)

Image tirée de Madlenka, de Peter Sis, paru chez Farrar Straus et Giroux,

(C) 2010 Peter Sis, avec permission de l'artiste. 
tats montrent que l'ensemble des professeurs semble d'accord pour accepter la langue des élèves en classe et aucun n'est réticent au fait de les utiliser en salle de classe.

Dans les observations de classe : davantage d'approches «plurielles» dans les classes expérimentales que dans les classes de contrôle, un effet de la formation et de l'utilisation des albums

Maintenant, si l'on croise les questionnaires aux observations de classe, on devrait naturellement observer des pratiques où les langues et les cultures sont sollicitées dans le cadre d'activités de classe et, dans tout type d'activité, ces langues et ces cultures devraient servir de ressources pour développer des compétences en français (lire le texte dans une langue connue avant de l'aborder en français, utiliser le dictionnaire ou une application multilingue, etc.). Or, les résultats montrent que les langues et les cultures sont davantage sollicitées dans les classes expérimentales. Ainsi, dans les classes de type contrôle, qui travaillent donc sans album, parler sur les langues et les cultures de la classe et les « autoriser» est très rare ( 2 enseignantes sur 6 ). Et pour les deux qui le pratiquent, les observations sont assez rarement dans un but de valorisation de langues des élèves, mais, davantage, pour terminer une séquence d'enseignement.

On voit donc ici un effet de la formation et de l'utilisation des albums comme facteurs favorisants l'utilisant de ces approches plurielles. Au-delà, on peut donc se poser la question de l'affirmation des enseignants des classes de contrôle à pratiquer ce type d'activité. Ces résultats semblent rejoindre une enquête menée en France (Auger et Cadet, 2016) où nous observions les mêmes distorsions entre le déclaratif et les pratiques de classe. Nous l'expliquons en France et au Canada par une injonction forte dans les textes officiels à reconnaître l'allophonie (en France) et le multiculturalisme (au Canada). Les enseignants de ces deux contextes craignent d'être discriminants, ils savent que les enfants migrants sont une population fragile, l'école vit sur l'idéologie de l'égalité/équité. Il semble donc important pour eux de dire que l'altérité est prise en compte. Mais dans les faits, faute de formation, cela n'est pas mis en pratique.

\section{Dans les observations de classe : des pratiques intégrant les langues et les cultures circonscrites à l'utilisation de l'album}

Un autre résultat est intéressant : dans les classes expérimentales, les approches plurielles sont davantage présentes que dans les groupes de type contrôle, mais elles sont essentiellement circonscrites au moment où l'enseignant utilise l'album de jeunesse. En effet, seulement un peu plus de la moitié des enseignants du groupe expérimental continue d'autoriser les langues et les cultures de la 
classe en dehors de ces moments (5/8 enseignants).

Il semble donc plus évident pour les enseignants de parler sur les langues et les cultures, que de les autoriser dans la classe. $\mathrm{Si}$, selon eux, il est plus facile de parler sur les langues et sur les cultures dans cette situation, c'est bien parce que l'album semble les fonder, les légitimer, les guider dans cette pratique. Comme annoncé dans le cadre conceptuel, on vérifie de nouveau que l'album est un vecteur intéressant et motivant où les langues et les cultures sont présentes.

\section{Des pratiques étayées par les entretiens}

Ces pratiques de classes sont étayées par les entretiens que nous avons pu mener conjointement aux observations de classes. Les discours des entretiens sont en contradiction avec les questionnaires dans lesquels tous les enseignants déclarent accueillir les langues et les cultures des enfants et les utiliser éventuellement comme une ressource pour les pratiques de classe. En revanche, ils sont bien en cohérence avec les pratiques de classes observées. En effet :

- Quatre enseignants sur huit, soit la moitié, se plaignent que les enfants parlent trop leur langue.

- Cinq enseignantes sur huit, soit plus de la moitié, remarquent que les enfants se traduisent trop entre eux.

Dans tous ces cas, et malgré nos sollicitations, aucune réflexion sur les opportunités d'utiliser les langues en tant que telles n'est formulée par les enseignants du groupe expérimental, malgré la formation et notre suivi pendant 12 semaines. Par ailleurs, tant chez les enseignants du groupe expérimental que chez ceux du groupe de contrôle, il y existe un malaise assez généralisé quand des éléments culturels religieux font irruption en classe. Cela s'explique en France par le principe de laïcité souvent réifié depuis les attentats, ainsi qu'à une absence de formation de la gestion de ce thème délicat. Au Canada, la situation minoritaire comme nous n'avons déjà souligné rend complexe la présence des autres langues.

\section{«Parler sur les langues et les cultures versus parler avec elles » : vers une redéfinition des approches plurielles et des pratiques translangagières}

Ainsi malgré la formation reçue pour le groupe expérimental, autoriser les langues et les cultures dans la salle de classe, par exemple pour traduire, comparer les langues, utiliser des supports multilingues comme les albums, ou des artefacts culturels divers comme des ouvrages multilingues, des dictionnaires, etc. qui sont des pratiques typiquement translangagières, sont encore un parent pauvre des pratiques pédagogiques. Il semble donc que, pour que le recours aux langues et aux cultures de la classe soit plus présent en classe, l' artefact de 
l'album doit être présent et utilisé comme un outil de premier plan. Il convient donc de le valoriser en tant que pratique. Le fait qu'il expose les élèves à différents scripts visuels et graphiques et à des thèmes liés à l'interculturel permet de dire qu'il incite à la réflexion sur ces sujets. Cependant, les enseignants ne s'autorisent pas encore à didactiser les langues et les cultures de la classe en dehors de ces moments consacrés à l'album.

Si l'on considère les albums donnés en exemple et les pratiques possibles, nous pouvons constater qu'ils permettent essentiellement de proposer de l'éveil aux langues, de l'intercompréhension et de l'interculturel. Par exemple, Tour du monde des bonnes manières permet de favoriser l'éveil aux langues sur les interactions de politesse, l'intercompréhension avec les différentes écritures et l'interculturel sur les universaux singuliers (Galisson, 1991) concernant les interactions sociales, L'oeuf du coq suggère l'éveil aux langues et l'intercompréhension phonologiques, Le magasin de mon père tout comme Madlenka sont des albums qui peuvent amener des réflexions sur l'éveil aux langues et sur l'interculturel en termes de phonologie, d'observation des expressions idiomatiques et leurs représentations interculturelles. Ce travail sollicite les expériences langagières et culturelles des élèves, mais pas à chaque fois, cela dépend des questions que pose l'enseignant et surtout des langues et cultures évoquées dans les albums. On remarque souvent que le professeur ne va pas toujours inclure les langues et cultures des enfants lors de ces séquences si elles ne sont pas déjà présentes dans l'album.

\section{Conclusion}

Selon nous, les approches plurielles visent une compétence «méta » sur les langues et les cultures, mais sans garanties que cette compétence soit développée en fonction des langues et cultures déjà présentes, même si les élèves peuvent se décentrer en projetant leurs expériences personnelles sur les langues et cultures qu'ils observent dans l'album. L'approche plurielle qui consiste en la didactique intégrée des langues et qui utilise des documents dans les langues des élèves est certainement la pratique qui se rapproche le plus de la définition du translanguaging, ce que Sierens et Van Avermaet (2014) englobent sous la dénomination «apprentissage multilingue fonctionnel » pour parler du volet pragmatique de l'utilisation concrète des langues dans la salle de classe. Finalement, nous pensons donc que les enseignants favorisent l'aspect «méta», donc le «parler sur» des approches plurielles quand ils les traduisent dans les activités translangagières qu'ils proposent aux élèves. Il convient donc de comprendre que «parler sur les langues et les cultures» n'implique pas forcément de parler «avec elles », ce qui est pourtant bien nécessaire psychoaffectivement et cognitivement. Pour intégrer aux pratiques translangagières les langues et les cultures des élèves, il convient d'abord de s'assurer qu'elles 
font bien partie du corpus de réflexion de la séquence de classe et que, si elles ne sont pas présentes dans l'album, il est nécessaire de les solliciter dans la discussion ou en ajoutant d'autres artefacts. Par ailleurs, pour ne pas créer un message contradictoire pour l'élève, ces pratiques ne doivent pas être oubliées, voire pire rejetées comme dans nos exemples, en dehors des moments où l'album n'est pas utilisé. C'est à partir de ces considérations ce que nous envisageons de mettre en œuvre nos nouvelles formations.

\section{Références}

Abdallah-Pretceille, M. (1995). Relations et apprentissage interculturels. Paris : Armand Colin.

Abdallah-Pretceille, M. et Porcher, L. (1998). Ethique de la diversité et éducation. Paris : Presses universitaires de France.

Association canadienne d'éducation de langue française (ACELF). (2006). Cadre d'orientation en construction identitaire: pour ouvrir un dialogue et élaborer ensemble notre vision. Québec : ACELF. www.acelf.ca/c/fichiers/ACELF_Cadre-orientation.pdf

Auger, N. (2005). Comparons nos langues : démarche d'apprentissage du français auprès d'enfants nouvellement arrivés (ENA). Montpellier, France : CRDP Languedoc-Roussillon. asl.univ-montp3.fr/masterFLE/n.auer/Livret_Comparons.pdf

Auger, N. (2010), Elèves nouvellement arrivés en France : réalités et perspectives en classe. Paris : Éditions des archives contemporaines.

Auger, N. (2017a). Developing competence for French as a foreign language within a plurilingual paradigm. Dans S. Coffey et U. Wingate (dir.). New directions in foreign language education research (p. 151-164). London, UK : Routledge.

Auger, N., (2017b). Frontières linguistiques dans les approches de la langue en théorie et en pratique : l'impact des contextes migratoires pour repenser l'enseignement en France. Dans S. Gorovitz (dir.), Frontières linguistiques en contextes migratoires. Citoyennetés en construction (p. 139-162). Paris : L'Harmattan.

Auger, N. et Cadet, L., (2016). Que révèlent les parcours et les pratiques des enseignants en classe de français? Éléments de réflexions sur les conflits cognitifs et méthodologiques face aux traditions didactiques. Dans P. Pégaz Paquet et L. Cadet (dir.), Les langues à l'école, la langue de l'école (p. 47-66). Artois, France : Artois Presses Universitare.

Beacco, J.-C. (2000). Les dimensions culturelles dans l'apprentissage des langues, des mots aux discours. Paris : Hachette.

Ben Kemoun, H. (2005). L'œuf du coq. Nantes, France : Éditions Casterman.

Berthiaume, D. (2004). L'observation de l'enfant en milieu éducatif. Montréal : Gaétan Morin.

Blanche-Benveniste, C. et Valli, A. (dir.). (1997). Le Français dans le Monde : Recherches et applications [numéro thématique : L'intercompréhension : le cas des langues romanes], janvier. 
Cadet, L., Mangiante J.-M., Laborde-Milaa, I. (2009). Langue(s) et intégration scolaire. Le français aujourd'hui, 164.

Candelier, M. (2008). Approches plurielles, didactiques du plurilinguisme : le même et l'autre. Recherches en didactique des langues et des cultures : les Cahiers de l'Acedle [numéro thématique : L'Alsace au cœur du plurilinguisme], 5, 65-90.

Cardinal, L. (1994). Ruptures et fragmentations de l'identité francophone en milieu minoritaire : un bilan critique. Sociologie et sociétés, 26, p. 71-86.

Cavalli, M. (2008). Didactique intégrée et approches plurielles. In C. Brohy, S. Resgui, (dir.), La didactique intégrée des langues : expériences et applications (p. 15-19). Genève, Suisse : Babylonia.

Cerquiglini, B. (1999). Les langues de la France. Rapport au ministre de l'Éducation nationale, de la Recherche et de la Technologie et à la ministre de la Culture et de la Communication. www.axl.cefan.ulaval.ca/francophonie/Rapport-Cerquiglini-1999. htm

Coste, D. et l'Association pour le dévelopement de l'enseignement bi-/plurilingue (ADEB) (dir.). (2013). Les langues au cour de l'éducation : principes, pratiques, propositions. Cortil-Wodon, Belgique : Éditions Modulaires Européennes (EME).

Cummins, J. (2013). Current research on language transfer : Implications for language teaching policy and practice. Dans P. Siemund, I. Gogolin, M.E. Schulz et J. Davydova (dir.), Multilingualism and language diversity in urban areas : Acquisition, identities, space, education (p. 289-304). Hambourg : John Benjamins.

Cuq, J.-P. (2003). Dictionnaire de didactique du français langue étrangère et seconde. Paris : CLE International.

Dupin de Saint-André, M. et Montesinos-Gelet, I. (2011). Grille pour étudier la qualité des oeuvres de littérature jeunesse : Quelques critères pour étudier la qualité des œuvres de littérature de jeunesse. diffusion-didactique.scedu.umontreal.ca:81/documents/ document.php?id=30

Escudé, P. et Janin, P. (2010). Le point sur l'intercompréhension, clé du plurilinguisme. Paris : CLE international.

Farmer, D. et Labrie, N. (2008). Immigration et francophonie dans les écoles ontariennes : comment se structurent les rapports entre les institutions, les parents et le monde communautaire? Revue des sciences de l'éducation, 34, 377-398. www. erudit.org/revue/rse/2008/v34/n2/019686ar.pdf

Ferréol, G. et Jucquois, G. (2003). Dictionnaire de l'altérité et des relations interculturelles. Paris : Armand Colin.

Fleuret, C. (2013). Quand la langue d'origine devient un levier nécessaire dans la résolution de problème orthographique chez des élèves en français langue seconde en difficultés d'apprentissage. Dans D. Daigle, I. Montesinos-Gelet et A. Plisson (dir.), Orthographe et populations exceptionnelles : perspectives didactiques (p. 81-104). Québec : Presses de l'Université du Québec. 
Fleuret, C. (sous presse). La littérature de jeunesse : une heuristique à la croisée des contextes d'apprentissage pour entendre la voix des élèves en situation minoritaire. Le Relais.

Fleuret, C., Bangou, F. et Ibrahim, A. (2013). Langues et enjeux interculturels : une exploration au cœur d'un programme d'appui à l'apprentissage du français de scolarisation pour les nouveaux arrivants. Revue canadienne de l'éducation, 36, p. 280298. journals.sfu.ca/cje/index.php/cje-rce/article/view/1558

Fleuret, C. et Sabatier, C. (2019). La littérature de jeunesse en contextes pluriels : perspectives interculturelles, enjeux didactiques et pratiques pédagogiques. Le Français dans le Monde : Recherches et applications [numéro thématique : Lectures de la littérature et appropriation des langues et cultures], 65, 95-111.

Fleuret, C. et Thibeault, J. (2016). Interactions verbales d'élèves allophones en retard scolaire lors de résolutions collaboratives de problèmes orthographiques [en ligne]. Recherches en didactique des langues et des cultures : les Cahiers de l'Acedle, 13. rdlc. revues.org/881

Forlot, G. (2009). L'anglais et le plurilinguisme. Pour une didactique des contacts et passerelles linguistiques. Paris : L'Harmattan.

Fournier, S. (2011). Le tour du monde des bonnes manières. Paris : Rue des enfants.

France. Ministère de l'éducation nationale et de la jeunesse (2012). Enseignements primaire et secondaire : scolarisation des élèves : organisation de la scolarité des élèves allophones nouvellement arrivés. Circulaire $\mathrm{n}^{0} 2012-141 \mathrm{du} 2-10-$ 2012. NOR : REDE1236612C. RED-DGESCO A1-1. www.education.gouv.fr/pid25535/ bulletin_officiel.html?cid_bo $=61536$

Galisson R. (1991). De la langue à la culture par les mots. Paris : CLE international.

García, O. et Wei, L. (2014). Translanguaging : Language, bilingualism, and education. New York : Palgrave MacMillan.

Goï, C. (2015). Des élèves venus d'ailleurs. Paris : Canopé.

Hawkins, E. (1984). Awareness of language : An introduction. Cambridge, UK : Cambridge University Press.

Héran, F. (2017). Avec l'immigration : mesure, débattre, agir. Paris : La Découverte.

Hornberger, N. (dir.). (2003). Continua of biliteracy : An ecological framework for educational policy, research and practice in multilingual settings. Clevedon, UK : Multilingual Matters.

Ichikawa, S. (2004). Le magasin de mon père. Paris : L'école des loisirs.

Institut national de la statistique et des études économiques (INSEE). (2013). Immigrés, étrangers. www.insee.fr/fr/statistiques/3633212

Johnson, R.B. et Onwuegbuzie, A.J. (2004). Mixed methods research : A research paradigm whose time has come. Educational Researcher, 33(7), 14-26. 
Kervran, M. (2006). Les langues du monde au quotidien : observation réfléchie des langues, cycle 2 . Rennes, France : Centre régional de documentation pédagogique (CRDP) de Rennes.

Ledent, J., Aman, C., Garnett, B., Murdoch, J., Walters, D. et McAndrew, M. 2013. Academic performance and educational pathways of young allophones : A comparative multivariate analysis of Montreal, Toronto and Vancouver. Canadian Studies in Population 40, p. 35-56.

McAndrew, M. et Ciceri, C. (2003). L'enseignement des langues d'origine au Canada : réalités et débats. Revue européenne des migrations internationales, 19, 173-194.

Moore, D. et Sabatier, C. (2014). Les approches plurielles et les livres plurilingues : de nouvelles ouvertures pour l'entrée dans l'écrit et pour favoriser le lien famille-école en milieu multilingue et multiculturel. Nouveaux Cahiers de la Recherche en Éducation [numéro thématique : Lire et écrire : les liens écolefamilles-communautés en contextes pluriels], 17(2), 32-65. www.erudit.org/fr/revues/ ncre/2014-v17-n2-ncre01902/1030887ar.pdf

Ontario. Ministère de l'Éducation. (2002). Le curriculum de l'Ontario de la 1re à la 8 e année : Actualisation linguistique en français [ALF] et Perfectionnement du français [PDF]. ottawa-ouest.cepeo.on.ca/wp-content/uploads/2018/08/ALF_perf.francais.pdf

Ontario. Ministère de l'Éducation. (2009). Une approche culturelle de l'enseignement pour l'appropriation de la culture dans les écoles de langue française de l'Ontario. www.edu.gov.on.ca/fre/amenagement/PourLaFrancophonie2009. pdf

Ontario. Ministère de l'Éducation. (2010a). Programme d'appui aux nouveaux arrivants [PANA] : le Curriculum de l'Ontario de la lre à la 8e année (révisé). www. edu.gov.on.ca/fre/curriculum/elementary/appui18curr.pdf

Ontario. Ministère de l'Éducation. (2010b). Actualisation linguistique en français [ALF] : le Curriculum de l'Ontario de la lre à la 8e année (révisé). www.edu.gov. on.ca/fre/curriculum/elementary/alf18curr2010.pdf

Roulet, E. (1980). Langue maternelle et langues secondes - vers une pédagogie intégrée. Paris : Hatier.

Schiff, C. (2012). En marge du métier, dispositifs d'intégration et pratiques enseignantes face aux élèves primo-migrants en collège. Dans C. Crenn et L. Kotobi (dir.), Du point de vue del'ethnicité : pratiques françaises (p. 111-124). Paris : Armand Colin.

Sierens, S. et Van Avermaet, P. (2014). Language diversity in education : Evolving from multilingual education to functional multilingual learning. Dans D. Little, C.Leung et P. Van Avermaet (dir.), Managing diversity in education : Languages, policies, pedagogies (p. 204-222). Clevedon, UK : Multilingual Matters.

Simon, D.-L., C. Dompmartin-Normand, S. Galligani et M.-O. Maire Sandoz (dir.). (2015). Accueillir l'enfant et ses langues : rencontres pluridisciplinaires sur le terrain de l'école. Paris : Riveneuve. 
Sis, P. (2000). Madlenka. Paris : Grasset Jeunesse.

Statistique Canada. (2009). Annuaire du Canada : Langues. No. du catalogue 11-402X. Ottawa. www150.statcan.gc.ca/n1/pub/11-402-x/2009/50000/cybac50000_000-fra.htm

Tauveron, C. (dir.) (2002). Lire la littérature à l'école : pourquoi et comment conduire cet apprentissage spécifique? De la GC au CM. Paris : Hatier.

Zarate, G. (1986). Enseigner une culture étrangère. Paris : Hachette.

Zarate, G. (1993). Représentation de l'étranger et didactique des langues. Paris : Didier. 


\section{Annexe 1: \\ La grille de Dupin de Saint-André et de Montesinos-Gelet (2011)}

\begin{tabular}{|c|c|c|c|}
\hline \multirow{6}{*}{$\begin{array}{l}\text { CONCEPTS } \\
\text { LITTÉRAIRES }\end{array}$} & \multicolumn{3}{|l|}{ L'œuvre est intéressante du point de vue : } \\
\hline & $\begin{array}{l}\text { - du paratexte (pages de couverture, titre, sous-titre, format de l'œuvre, matière } \\
\text { des pages, pages de garde, page titre, avertissement, dédicace, table des matières, } \\
\text { pagination) }\end{array}$ & Oui & Non \\
\hline & $\begin{array}{l}\text { - du système des personnages (personnalité des personnages, caractérisation directe ou } \\
\text { indirecte, relation entre les différents personnages, évolution des personnages, personnage } \\
\text { stéréotype de la littérature de jeunesse, etc.) }\end{array}$ & Oui & Non \\
\hline & $\begin{array}{l}\text { - du système énonciatifi (focalisation [point de vue interne, externe ou omniscient], variation } \\
\text { des points de vue, narrateur qui interpelle le lecteur) }\end{array}$ & Oui & Non \\
\hline & - du traitement de l'espace et du temps & Oui & Non \\
\hline & - de l'intertextualité & Oui & Non \\
\hline \multirow{7}{*}{$\begin{array}{l}\text { LES SIX TRAITS } \\
\text { D'ÉCRITURE }\end{array}$} & \multicolumn{3}{|l|}{ L'œuvre est intéressante du point de vue : } \\
\hline & - des idées & Oui & Non \\
\hline & $\begin{array}{c}\text { - de la structure du texte (linéaire, enchâssée, répétitive, en boucle, en parallèle, } \\
\text { perturbation de l'ordre chronologique, introduction, chute) et de l'œuvre }\end{array}$ & Oui & Non \\
\hline & $\begin{array}{l}\text { - du rythme et de la syntaxe (structure et longueur des phrases, début des phrases, } \\
\text { I'enchaînement des phrases) }\end{array}$ & Oui & Non \\
\hline & $\begin{array}{l}\text { - du choix des mots (mots précis, mots évocateurs, onomatopées, rimes, variété lexicale, } \\
\text { registres de langue, etc.) }\end{array}$ & Oui & Non \\
\hline & - de la voix (le style de l'auteur, les figures de style) & Oui & Non \\
\hline & - des conventions linguistiques (orthographe, grammaire, ponctuation) & Oui & Non \\
\hline \multirow{10}{*}{ ILLUSTRATION } & \multicolumn{3}{|l|}{ Les illustrations sont intéressantes du point de vue } \\
\hline & - de la mise en pages (dissociation, association, compartimentage, conjonction) iv $^{\mathrm{v}}$ & Oui & Non \\
\hline & - de la narration visuelle & Oui & Non \\
\hline & $\begin{array}{l}\text { - du cadrage (choix des plans [ensemble, général, moyen, américain, rapproché, gros plan et } \\
\text { très gros plan] et des angles de vue [normal, plongée, contre-plongée, en visée subjective] } \\
\text { v) et du décadrage }\end{array}$ & Oui & Non \\
\hline & - du cadre (forme, bordure du cadre, sortie de cadre, surcadrage) ${ }^{\text {vii }}$ & Oui & Non \\
\hline & - du choix des couleurs (chaudes, froides, contraste) & Oui & Non \\
\hline & - du montage & Oui & Non \\
\hline & - de l'esthétisme ${ }^{\mathrm{ix}}$ & Oui & Non \\
\hline & - du traitement du temps et de l'espace & Oui & Non \\
\hline & - de l'intericonicité & Oui & Non \\
\hline $\begin{array}{l}\text { RAPPORT } \\
\text { TEXTE/IMAGE }\end{array}$ & $\begin{array}{l}\text { Le rapport texte-image est original }{ }^{x} \text { : } \\
\text { - même si le texte et l'image sont redondants } \\
\text { - le texte et l'image « collaborent » } \\
\text { - le texte et l'image « s'opposent » }\end{array}$ & $\begin{array}{l}\text { Oui } \\
\text { Oui } \\
\text { Oui }\end{array}$ & $\begin{array}{l}\text { Non } \\
\text { Non } \\
\text { Non }\end{array}$ \\
\hline \multirow[t]{5}{*}{$\begin{array}{l}\text { INTÉRÊTS } \\
\text { PÉDAGOGIQUES } \\
\text { DE L'OEUVRE }\end{array}$} & 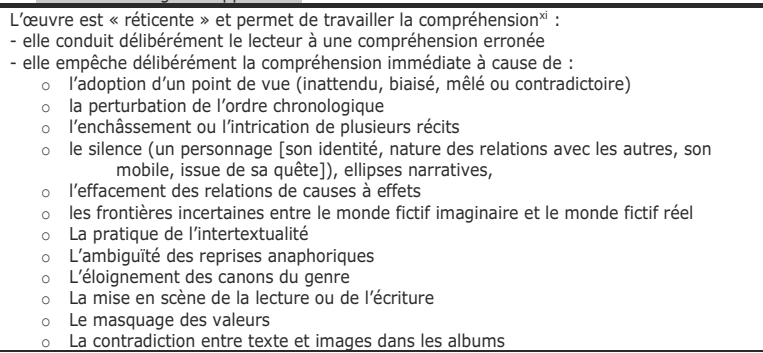 & $\begin{array}{l}\text { Oui } \\
\text { Oui } \\
\text { Oui } \\
\text { Oui } \\
\text { Oui } \\
\text { Oui } \\
\text { Oui } \\
\text { Oui } \\
\text { Oui } \\
\text { Oui } \\
\text { Oui } \\
\text { Oui } \\
\text { Oui }\end{array}$ & $\begin{array}{l}\text { Non } \\
\text { Non } \\
\text { Non } \\
\text { Non } \\
\text { Non } \\
\\
\text { Non } \\
\text { Non } \\
\text { Non } \\
\text { Non } \\
\text { Non } \\
\text { Non } \\
\text { Non } \\
\text { Non }\end{array}$ \\
\hline & L'œuvre est « proliférante » et permet de travailler l'interprétation ${ }^{\text {xii }}$ & Oui & Non \\
\hline & L'œuvre est propice à la mise à place d'un projet d'écriture ${ }^{\text {xiii }}$ & Oui & Non \\
\hline & L'œuvre est propice à une lecture interactive & Oui & Non \\
\hline & $\begin{array}{l}\text { L'œuvre se prête bien à l'intégration des matières (ex. : éthique et culture religieuse, univers } \\
\text { social, sciences, etc.) }\end{array}$ & Oui & Non \\
\hline \multirow{8}{*}{$\begin{array}{l}\text { RECONNAISSANCE } \\
\text { DE L'CEUVRE }\end{array}$} & L'œuvre a été primée & Oui & Non \\
\hline & L'œuvre est recommandée sur le site « livres ouverts » du MELS & Oui & Non \\
\hline & L'œuvre est recommandée sur le site « Ricochet » & Oui & Non \\
\hline & L'œuvre est recommandée sur le site « Sentiers littéraires » & Oui & Non \\
\hline & L'œuvre fait partie du palmarès de Communication Jeunesse & Oui & Non \\
\hline & L'œuvre est recommandée dans la revue « Lurelu » & Oui & Non \\
\hline & L'œuvre est recommandée dans « la revue des livres pour enfants » & Oui & Non \\
\hline & L'œuvre est recommandée dans la revue « Le pollen » & Oui & Non \\
\hline
\end{tabular}

Tableau reproduit avec la permission des auteurs, M. Dupin de Saint-André et I. Montésinos-Gelet. 


\section{Notes}

i Tous les critères de la catégorie « concepts littéraires », excepté celui du paratexte (Tsimbidy, 2008) sont extraits de la grille d'analyse de Couet-Butlen (2007).

ii Les éléments grisés sont des critères qui peuvent constituer des facteurs de résistance d'une oeuvre.

iii Critères d'Olness (2005).

iv Catégorisation des mises en pages de Van der Linden (2007).

v Catégorisation du cadrage de Roux (1994) et de Van der Linden (2007).

vi Ce critère est proposé par Van der Linden (2007).

vii Ces critères sont proposés par Van der Linden (2007). viii Ce critère est proposé par Van der Linden (2007).

ix Ce critère est proposé par Couet-Butlen (2007).

$\mathrm{x}$ Catégorisation du rapport texte-image de Van der Linden (2007).

xi Tous les critères qui suivent sont extraits de Tauveron (1999).

xii C'est « un texte ouvert présentant de nombreux éléments potentiellement polysémiques, des indices pouvant entrer dans plusieurs réseaux et donc diversement interprétables » (Tauveron, 1999, p. 20).

xiii Ce critère est proposé par Couet-Butlen (2007).

\section{Références}

Couet-Butlen, M. (2007). Des critères de choix des ouvrages et des pratiques de lecture à l'école. Paris : CRDP de l'académie de Créteil. Repéré à : www.cndp.fr/crdp-creteil/telemaque/ document/choixouvrages.htm

Ollness, R. (2005). Using literature to enhance writing instruction. Newark, DE : International Reading Association.

Roux, P. (1994). La BD : 1'art d'en faire. Ottawa : Centre Franco-Ontarien de ressources pédagogiques.

Tauveron, C. (1999). Comprendre et interpréter le littéraire à l'école : du texte réticent au texte proliférant. Repères, 19, 9-38.

Tsimbidy, M. (2008). Enseigner la littérature de jeunesse. Toulouse, France : Presses universitaires du Mirail.

Van der Linden, S. (2007). Lire l'album. Puy-en-Velay : L'atelier du poisson soluble. 


\section{Annexe 2: \\ Le questionnaire ${ }^{9}$}

Nous vous remercions de bien vouloir prendre un peu de votre temps pour répondre au questionnaire. Vos réponses demeureront confidentielles. Vous n'êtes pas obligé de répondre à toutes les questions. Une fois terminé, veuillez le remettre dans l'enveloppe fournie.

1. Depuis combien de temps enseignez-vous?

$\square$ Moins de 5 ans

$\square 5$ à 10 ans

๑ 10 à 15 ans

$\square$ Plus de 15 ans

2. Depuis combien de temps enseignez-vous aux élèves en ALF (Canada) aux EANA (France) ? $\square$ Moins de 5 ans

$\square$ à 10 ans

$\square 10$ à 15 ans

$\square$ Plus de 15 ans

3. Qu'est-ce qui vous a conduit à travailler avec ces élèves ?

$\square$ choix personnel

$\square$ choix administratif

$\square$ autres Lequel?

Pourquoi?

4. Quelle(s) langue(s) parlez-vous au quotidien ?

$\square$
$\square$
$\square$
$\square$
0

5. Comment les connaissez-vous? (Plusieurs réponses possibles — en face des réponses, écrivez vos langues)

$\square$ par ma famille

$\square$ par mon environnement

\footnotetext{
${ }^{9}$ Ce questionnaire est un instrument qui a été construit par les auteures (Fleuret et Auger) dans le cadre du projet "Pour une didactique interculturelle renouvelée du français langue seconde et vers une meilleure réussite scolaire des éléves allophones : une comparaison intersite France-Canada," financé par le Conseil de recherche des sciences humaines du Canada (CRSH, 2016-2019).
} 
$\square$ par mes études

$\square$ par mes voyages

$\square$ autres langues que vous connaissez, mais que vous n'utilisez pas en ce moment

Lesquelles?

6. Quel est votre niveau EN ANGLAIS ?

\begin{tabular}{|c|l|l|l|l|l|}
\hline & & Faible & Assez bon & bon & Très bon \\
\hline À l'oral & & & & & \\
\hline À l'écrit & Écriture & & & & \\
\hline À l'écrit & Lecture & & & & \\
\hline
\end{tabular}

7. Quel est votre niveau pour votre AUTRE LANGUE? (La nommer :

\begin{tabular}{|c|l|l|l|l|l|}
\hline & & Faible & Assez bon & bon & Très bon \\
\hline À l'oral & & & & & \\
\hline À l'écrit & Écriture & & & & \\
\hline À l'écrit & Lecture & & & & \\
\hline
\end{tabular}

8. Quel est votre niveau pour votre AUTRE LANGUE? (La nommer :

\begin{tabular}{|l|l|l|l|l|l|}
\hline & & Faible & Assez bon & bon & Très bon \\
\hline À l'oral & & & & & \\
\hline À l'écrit & Écriture & & & & \\
\hline À l'écrit & Lecture & & & & \\
\hline
\end{tabular}

9. Quel est votre niveau pour votre AUTRE LANGUE ? (La nommer :

\begin{tabular}{|l|l|l|l|l|l|}
\hline & & Faible & Assez bon & bon & Très bon \\
\hline À l'oral & & & & & \\
\hline À l'écrit & Écriture & & & & \\
\hline À l'écrit & Lecture & & & & \\
\hline
\end{tabular}

10. Quel est votre niveau pour votre AUTRE LANGUE? (La nommer :

\begin{tabular}{|l|l|l|l|l|l|}
\hline & & Faible & Assez bon & bon & Très bon \\
\hline À l'oral & & & & & \\
\hline À l'écrit & Écriture & & & & \\
\hline À l'écrit & Lecture & & & & \\
\hline
\end{tabular}


11. Sur une échelle de 1 à 5 , vous sentez vous «éloigné » (1) ou «proche » (5) de ces langues :
$\square$ anglais
$\square$ arabe
$\square$ espagnol
$\square$ occitan
$\square$ portugais
$\square$ roumain
$\square$ chinois
$\square$ créole
$\square$ ukrainien
$\square$ farsi
$\square$ autres (lesquelles?)

12. De quelle conception générale d'apprentissage des langues vous sentez-vous le plus proche :

$\checkmark$ «On apprend la langue en connaissant d'abord le code (les règles de grammaire, d'orthographe ...)»

$\square$ «On apprend le code en connaissant d'abord la langue (parler, échanger, reformuler, lire ...)»

Expliquez brièvement votre choix :

13. Comment repérez-vous ces compétences de langue ? (Plusieurs réponses possibles)

$\square$ vous connaissez les familles des élèves

$\square$ par les évaluations du CASNAV

$\square$ vous reconnaissez les langues

$\square$ vous connaissez les langues des élèves

$\square$ les élèves parlent parfois ces langues, ou des bribes de langue, de manière consciente en classe

$\square$ les élèves mêlent, dans le français de la classe, des mots, des expressions, qui ne sont pas du français enseigné en classe

14. De quelle pratique vous sentez-vous le plus proche :

$\square$ «je préfère ne pas trop faire référence aux expériences vécues en dehors de l'école. » Expliquez les raisons :

凹 «je suis à l'aise pour inciter l'élève à s'appuyer sur les expériences vécues à la maison. » Expliquez les raisons : 
15. Selon vous, est-ce que vos élèves ont des compétences en langue autre que française? (Plusieurs réponses possibles)

$\square$ non

$\square$ oui

$\square$ ce ne sont pas des compétences réutilisables pour la classe

$\square$ ce sont des compétences qu'ils ont des difficultés à «maitriser »

$\square$ c'est moi qui suis l'enseignant ${ }^{10}$ et c'est difficile pour moi d'étayer avec ces compétences

et donc d'aider l'élève

$\square$ je peux étayer avec ces compétences

$\square$ c'est eux qui s'étayent entre eux avec leurs compétences

16. Faites-vous en classe des liens entre le français (forme d'un mot, sens d'un mot ...) et d'autres langues? (Plusieurs réponses possibles)

$\square$ non

$\square$ parfois

$\square$ souvent

$\square$ je voudrais, mais je n'en ai pas la compétence

$\square$ cela ne sert à rien

$\square$ cela n'intéresse pas les élèves

$\square$ cela intéresse les élèves

$\square$ cela fait progresser les élèves

$\square$ on ne parle que français dans la classe, car c'est la politique scolaire

$\square$ si on veut que les élèves apprennent le français, on doit leur parler français à l'école

17. Si un élève ne comprend pas un texte littéraire ou un extrait de journal, l'encouragez-vous à trouver le support dans les langues qu'il connaît déjà ?

$\square$ oui

$\square$ non

18. Trouvez-vous cela rentable, intéressant pédagogiquement?

$\square$ oui

$\square$ non

19. Avez-vous déjà tenté une telle pratique?

$\square$ Oui

a Non

Si oui avec quels résultats?

${ }^{10}$ Le masculin est utilisé dans le seul but d'alléger le texte. 
20. Connaissez-vous les textes suivants et seriez vous capables de résumer succinctement leur contenu ? (oui/non)

\section{Canada :}

$\square$ Loi sur le multiculturalisme

$\square$ la résumer oui/non

$\square$ Politique d'aménagement linguistique (PAL)

$\square$ la résumer oui/non

$\square$ Programme d'appui aux nouveaux arrivants (PANA)

$\square$ le résumer oui/non

$\square$ Programme d'actualisation linguistique en français (ALF)

$\square$ le résumer oui/non

$\square$ Cadre Européen Commun de Référence pour les Langues

$\square$ le résumer oui/non

Pour les items où vous avez répondu oui, pouvez-vous expliquer les grandes lignes de chacun en nommant le texte en question

Quel impact cela a-t-il eu sur vos pratiques?

\section{France}

$\square$ Cadre Européen Commun de Référence pour les Langues

$\square$ le résumer oui/non

$\square$ La circulaire de 2012 sur la scolarisation des EANA

a la résumer oui/non

$\square$ Le socle commun

$\square$ Les nouveaux programmes de l'École primaire

$\square$ Loi de refondation de l'école de la république de 2012 (notamment la partie concernant le français, les langues vivantes régionales, étrangères, de la famille)

$\square$ la résumer oui/non

Pour les items où vous avez répondu oui, pouvez-vous expliquer les grandes lignes de chacun en nommant le texte en question 
Quel impact cela a-t-il eu sur vos pratiques?

21. Comment prenez-vous en compte la diversité culturelle dans vos pratiques pédagogiques? (Plusieurs réponses possibles)

$\square$ en prenant appui sur les programmes scolaires

$\square$ je vais chercher des ressources sur les pays connus/où ont vécu les élèves

Comment?

Quelles ressources?

$\square$ je fais venir les parents dans ma classe pour une activité spécifique

Laquelle?

À quelle fréquence?

$\square$ j'essaie de parler quelques mots dans la ou les langues des familles

Lesquels?

Pourquoi?

Dans quel contexte?

$\square$ j'utilise des livres qui évoquent les pays, les langues ou les cultures connus/ où ont vécu les élèves

Conçus en France?

Conçus au Canada?

Conçus dans le pays des élèves?

$\square$ je les encourage à écrire/ à parler de ces pays

$\square$ autres (lesquelles ?)

22. Comment être certain qu'un élève a bien compris un texte ? (Plusieurs réponses possibles) $\square$ par la lecture à haute voix que je fais sur le texte que je propose

$\square$ par un questionnaire à choix multiples

$\square$ par des questions ouvertes à l'oral

$\square$ par des questions ouvertes à l'écrit

$\square$ par des questions fermées à l'écrit

23. Selon vous, les termes « erreur » et « faute»

$\square$ sont équivalents

$\square$ ne sont pas équivalents

$\square$ ce n'est pas important

$\square$ j'utilise plus le terme faute dans ma classe et pour la correction des travaux

$\square$ j'utilise plus le terme erreur dans ma classe et pour la correction des travaux 
24. Un élève arrive de Roumanie, un second de Londres ou de Régina sans parler français. Vous pensez que :

$\square$ «Ce sera plus simple de développer des compétences en français pour l'élève arrivant de Roumanie »

$\square$ «Ce sera plus simple de développer des compétences en français pour l'élève arrivant de Londres ou de Régina »

$\checkmark$ Ni l'un ni l'autre

Pourquoi?

25. Quels sont les supports écrits les plus courants que vous utilisez en lecture?

$\square$ littérature de jeunesse (Lesquels ?)

$\square$ manuel scolaire (Lesquels ?)

$\square$ journaux (Lesquels ?)

$\square$ prospectus

$\square$ magazines (Lesquels ?)

$\square$ autres (Lesquels ?)

26. Quelles sont les pratiques pédagogiques que vous utilisez le plus en lecture ?

$\square$ lecture en dyade

$\square$ lecture autonome

$\square$ lecture en groupe

$\square$ lecture à voix haute

$\square$ autres (Lesquelles?)

27. Quelles sont les pratiques pédagogiques que vous utilisez le plus en écriture ? (plusieurs réponses possibles)

$\square$ travail d'équipe

$\square$ travail personnel

$\square$ dyades

$\square$ trios

$\square$ orthographes approchées

$\square$ autres (Lesquelles?) 